\title{
Hospitalization Costs of Respiratory Diseases Attributable to Temperature in the Context of Climate Change in Australia
}

\section{Michael Tong}

The University of Adelaide - North Terrace Campus: The University of Adelaide https://orcid.org/0000-0002-9694-9207

\section{Berhanu Wondmagegn}

The University of Adelaide

\section{Jianjun Xiang}

The University of Adelaide

\section{Susan Williams}

The University of Adelaide

\section{Alana Hansen}

The University of Adelaide

\section{Keith Dear}

The University of Adelaide

\section{Dino Pisaniello}

The University of Adelaide

\section{Blesson Varghese}

The University of Adelaide

\section{Jianguo Xiao}

WA Health: Government of Western Australia Department of Health

\section{Le Jian}

WA Health: Government of Western Australia Department of Health

\section{Ben Scalley}

WA Health: Government of Western Australia Department of Health

\section{Monika Nitschke}

SA Health

John Nairn

Australian Bureau of Meteorology

Hilary Bambrick

Queensland University of Technology - QUT: Queensland University of Technology

Jonathan Karnon

Flinders University

Peng Bi ( $\sim$ peng.bi@adelaide.edu.au )

The University of Adelaide - North Terrace Campus: The University of Adelaide https://orcid.org/0000-0002-3238-3427

\section{Research}

Keywords: Hospitalization cost, Respiratory diseases, Temperature, Climate Change

Posted Date: December 21st, 2021

DOI: https://doi.org/10.21203/rs.3.rs-1140619/v1

License: (a) (1) This work is licensed under a Creative Commons Attribution 4.0 International License. Read Full License 


\section{Abstract}

\section{Background:}

The association between temperatures and respiratory diseases has been extensively reported. However, the associated healthcare costs and attributable fractions due to temperature have scarcely been explored. The aims of this study were to estimate respiratory disease hospitalization costs attributable to non-optimum ambient temperature, to quantify the attributable fraction from cold and hot temperatures, and to estimate the future hospitalization costs in two Australian cities.

\section{Methods:}

The associations between daily hospitalization costs for respiratory diseases and temperatures in Sydney and Perth over the study period of 2010-2016 were analyzed using distributed lag non-linear models. Future hospitalization costs for respiratory diseases were estimated based on three predicted climate change scenarios - RCP2.6, RCP4.5 and RCP8.5.

\section{Results:}

The estimated respiratory disease hospitalization costs attributable to non-optimum ambient temperatures increased from 493.2 million Australian dollars (AUD) in 2010s to more than 700 million AUD in 2050s in Sydney, and from 98.0 million AUD to about 150 million AUD during the same period in Perth, in large part due to population growth. In the context of climate change, the current cold attributable fraction in Sydney (23.7\%) and Perth (11.2\%) is estimated to decline by the middle of this century to (18.1-20.1\%) and (5.1$6.6 \%$ ) respectively, while the heat-attributable fraction for respiratory disease is expected to gradually increase from $2.6 \%$ up to $5.5 \%$ in Perth.

\section{Conclusions:}

This study found both cold and hot temperatures increased the overall hospitalization costs for respiratory diseases in two major Australian cities, although the attributable fractions varied. The largest contributor was cold temperatures. While respiratory disease hospitalization costs will increase in the future, climate change will result in a decrease in the cold attributable fraction and an increase in the heat attributable fraction, depending on the location.

\section{Background}

Global temperature has risen markedly since industrialization began around 1750 largely due to anthropogenic activities and the increases in greenhouse gas emissions, e.g. from energy production, transportation, agriculture and industries [1]. The increasing temperature and climate change are the greatest threat to human health in history [2]. The impact of climate change and its impact on population health has been explored extensively over the past two decades [3]. Many epidemiological studies have demonstrated the negative impacts of climate change on population health [4], and growing evidence has shown increasing temperatures were associated with increased morbidity and mortality of a range of temperature-sensitive diseases, including direct heat associated diseases, e.g., heatstroke, cardiovascular diseases, renal diseases, mental health disorders, and indirect vector-borne infectious diseases, e.g., dengue fever, malaria, ross river virus, and hemorrhagic fever with renal syndrome [4-13]. However, it should be noted that cold temperatures can also be associated with poor health outcomes, and cold-related diseases should not be overlooked because of the recent focus on heat-related diseases in the context of climate change. One of the typical disease categories that is associated with cold temperatures is respiratory disease $[14,15]$. Studies across the world in different climatic conditions and geographical zones have almost reached a consensus that respiratory diseases are more likely to occur during the winter period or in low-temperature conditions [14-17]. Nevertheless, the associated economic burden to the healthcare system has not been well explored. Climate change has further added urgency to better understand the economic burden of diseases not only due to increasing temperatures, but also possible reduced effects due to cold temperatures.

Studies have found both hot and cold temperature exposure can increase the risk of respiratory diseases $[15,18,19]$. A study in the USA indicated each degree above a threshold of daily mean temperature $28.9{ }^{\circ} \mathrm{C}$ was associated with a $2.7 \%-3.1 \%$ increase in the same-day hospitalizations due to respiratory diseases [18]. In Europe, a study conducted among 12 cities found $1{ }^{\circ} \mathrm{C}$ increase in daily maximum temperature was associated with $1.2 \%-2.1 \%$ increase in respiratory admissions within lag 0-3 days [19]. By contrast, a study in Finland found $1{ }^{\circ} \mathrm{C}$ decrease in average temperature increased the risks for upper respiratory tract infections by $4.3 \%$, and lower 
respiratory tract infections by $2.1 \%$ [15]. Moreover, studies in Australia and China showed both hot and cold temperatures increase the risk of respiratory diseases $[16,17]$, and several other studies also indicated that a large temperature change significantly increased the risk of respiratory diseases $[20,21]$. Although previous studies have found a significant association between ambient temperature exposures and respiratory diseases, very limited studies have assessed the healthcare costs attributable to ambient temperatures, nor estimated the future healthcare costs for respiratory diseases in the context of climate change $[22,23]$.

In Australia, it is predicted that the annual average temperature will increase by $0.6-1.3^{\circ} \mathrm{C}$ in the $2030 \mathrm{~s}$ above the reference period of 1986-2005, and by up to $2.8-5.1^{\circ} \mathrm{C}$ at the end of century [24]. Such temperature change could further impact the incidence of respiratory diseases, and increase or decrease the associated economic burden to healthcare in this region.

The present study aimed to examine respiratory disease hospitalization costs attributable to non-optimum ambient temperature; quantify the attributable fractions from hot and cold temperatures; and estimate the associated future hospitalization costs in two Australian cities - Sydney and Perth - in the context of climate change. The results will help local and regional government, health authorities and communities to have a better understanding of climate change attributable hospitalization costs; to plan, evaluate and optimize current climate change adaptation strategies and better direct medical resources to respond to respiratory diseases in the context of climate change.

\section{Methods}

\subsection{Study setting}

This study was conducted in two cities of Australia - Sydney and Perth. Sydney is located on Australia's east coast and has a temperate climate with warm summers and cool winters. It is the largest city of Australia with an estimated population of 5.5 million in 2020 , living in an area of 12,368 square kilometres [25]. Perth, about 4,000 kilometers away from Sydney, is located on Australia's west coast and has a Mediterranean climate with hot and dry summers, cool and wet winters. It is the fourth largest city in Australia with an estimated population of 2.1 million in 2020, living in a metropolitan area of 6,418 square kilometres [25]. These cities were selected to evaluate the respiratory disease hospitalization costs in different demographic and climatic contexts in Australia [26]. Three time periods were used in this study: 2010s, 2030s and 2050s. The baseline period of July 2010-June 2016 was defined as 2010s; the future corresponding periods 2030-2036 and 2050-5056 were defined as 2030s and 2050s, respectively.

\subsection{Data sources}

\subsubsection{Hospitalisation cost data}

Respiratory disease hospitalization costs: Daily respiratory disease (ICD-10-AM: J00-J99) hospital admissions and associated hospitalization costs from public hospitals over the study period from July 2010 to June 2016 in Sydney and Perth were obtained from the Australian National Independent Hospital Pricing Authority (IHPA). The daily respiratory disease hospitalization costs were aggregated by the date of admission for statistical analysis.

\subsubsection{Meteorological data}

Meteorological data: Daily minimum (Tmin) and maximum (Tmax) temperatures were obtained from the Australian Bureau of Meteorology. Daily mean (Tmean) temperature was calculated by the average of Tmin and Tmax. Tmin, Tmean and Tmax are the averages calculated from 17 weather observation stations in Sydney, and 13 weather observation stations in Perth (Figure S1). Daily mean temperatures for the 2030s and 2050s were projected based on three Commonwealth Scientific and Industrial Research Organisation (CSIRO)- defined Representative Concentration Pathways (RCPs). Depending on the RCP emission scenarios, the temperature in Sydney is projected to increase $0.9-1.2^{\circ} \mathrm{C}$ for the period $2030 \mathrm{~s}$, relative to the reference period 1986-2005; and by 1.0$2.0^{\circ} \mathrm{C}$ for the period 2050s (Table S1) [27]. In Perth, the temperature is projected to increase $0.8-1.0^{\circ} \mathrm{C}$ by the 2030 s, and by $0.9-1.8^{\circ} \mathrm{C}$ for the period 2050s, relative to the reference period 1986-2005 [27].

Page $3 / 16$ 
In addition, population data for the baseline period 2010s and projection periods 2030s and 2050s were obtained from the Australian Bureau of Statistics (ABS) for the two cities [25]. For the projection periods we adopted the ABS 'medium' levels of fertility, life expectancy, net overseas migration and interstate flows for the two cities. Daily populations were estimated using the linear interpolation method [28].

\subsection{Statistical analyses}

The methods for statistical analyses had been described in our previous studies on assessing the effects of temperature on healthcare costs in Adelaide, Perth and Sydney [29-31]. Two-stage data analyses were performed to explore the association between temperature and hospitalization costs and predict future hospitalization costs. In the first stage, the relationships between daily mean temperature and daily respiratory disease hospitalization costs were estimated using a generalized linear time series regression with a distributed lag non-linear model (DLNM) [32]. In the second stage, the future temperature-attributable respiratory disease hospitalization costs were estimated based on the baseline associations, projected temperatures, and populations for 2030s and 2050s.

\subsubsection{First stage}

To assess the shape of the exposure-lag-response relationship, a DLNM model with Gamma distribution was fitted simultaneously to estimate the possible non-linear relationship and lagged effects of temperature on hospitalization costs [32-34]. The model controlled for long-term trends, seasonality, weekday variations and public holidays. The exposure-response curves were modelled using a natural cubic spline for temperature with three internal knots placed at the 10th, 75th, and 90th percentiles of temperature distributions, and the lag-response curves with a natural cubic spline with an intercept and three internal knots placed at equally spaced values in the log scale $[35,36]$. To control for location-specific seasonality and long-term trends, a B-spline with 8 degrees of freedom ( $d f$ ) per year for time [bs(time,8df per year $\times 6$ years)] in Sydney and $4 d f$ per year for time [bs(time, $4 d f$ per year $\times 6$ years)] in Perth were included in our models [34]. To control for weekday variations, the day of week (dow) was also included in the model. Public holidays (pubhol) were controlled by using a binary variable. The temperature effects were calculated relative to the optimum temperature (i.e. the temperature at which the minimum relative risk for hospitalization costs occurred), which was obtained from the cumulative exposureresponse curve for respiratory disease hospitalization costs, as per the methods of Gasparini et al. [33, 37] The location-specific models were described as follows:

$$
\begin{aligned}
& \text { InSydney }: \log \left[\mathrm{E}\left(Y_{t}\right)\right]=\alpha+c b\left(\text { Temp }_{t}\right)+b s(\text { time, 8dfperyear } \times 6 \text { years })+\text { dow }_{t}+\text { pubhol }_{t} \\
& \text { In Perth: } \log \left[\mathrm{E}\left(Y_{t}\right)\right]=\alpha+c b\left(\text { Temp }_{t}\right)+b s(\text { time, } 4 \text { dfperyear } \times 6 \text { years })+\text { dow }_{t}+\text { pubhol }_{t}
\end{aligned}
$$

Where,

$Y_{t}$ is respiratory disease hospitalization costs on day $\mathrm{t}$;

a is the intercept;

$c b\left(\operatorname{Temp}_{t}\right)$ is the cross-basis natural cubic spline function for daily mean temperature with both response and lag dimension applied from the DLNM;

bs(time, 8 and 4 df per year $\times 6$ years) is the B-spline with degrees of freedom per year multiplied by the 6 year study period, adjusted for seasonality and long-term trend in Sydney and Perth [38],

time is in days;

dow is the day of week on day $t$, and

pubhol is a binary variable representing public holidays on day $t$.

The optimum temperature for respiratory disease hospitalization costs was identified via an overall cumulative exposure-response curve in the first stage, and the outcome below or above the optimum temperature was assigned as the temperature effect due to

Page $4 / 16$ 
exposures [32,34]. The daily mean temperature was chosen as the index providing the best fit in the analysis of respiratory disease hospitalization costs. In order to completely capture the temperature-related hospitalization costs, a maximum of 14 lag days was used in the model, and the value showing the best fit for the respiratory diseases in this study. We tested these modelling choices in sensitivity analysis, which was conducted by changing temperature metrics from daily mean to minimum and maximum temperatures, $d f$ for time from $4 d f$ to $8 d f$ per year, and the maximum lag days of 14 days to 7-21 days, to compare and best capture the effects of temperature on hospitalisation costs. Residual analysis and autocorrelation tests were conducted to evaluate the goodness of model fit and autocorrelation. To ensure the hospitalization costs were comparable across the different years, consumer price index (CPI) data were obtained from the Australian Bureau of Statistics [39], and the daily hospitalization costs were adjusted for inflation and standardized to the second quarter of 2016 in Australian dollars (AUD).

\subsubsection{Second stage}

After the baseline exposure-response relationships between daily temperatures and hospitalization costs were obtained from the first stage analysis, future respiratory disease hospitalization costs were estimated based on baseline model and projected temperature changes in 2030s and 2050s. The future temperature effects on hospitalization costs were estimated under three temperature scenarios (RCP2.6, RCP4.5, and RCP8.5 emission scenarios) for the periods 2030s and 2050s. Hospitalization costs and fractions attributable to temperature with reference to the optimum temperature of minimum relative risk were calculated to show temperature attributable costs (AC) and attributable fractions (AF). The AC and AF were calculated using a method of Gasparrini and Leone [35]. The $A C$ and $A F$ are defined as:

$$
\begin{gathered}
A C_{X, t}=A F_{X, t} \times C_{t} \\
A F_{x, t}=1-\exp \left(-\sum_{l=0}^{L} \beta_{x_{t-l}, l}\right)
\end{gathered}
$$

Where,

$x$ is the daily mean temperature exposure on day $t$,

$C_{t}$ is the daily hospitalisation costs on day $t$.

$\beta_{x_{t-l}, l}$ is the natural logarithm of RR given exposure on day $t-/$ after / days have elapsed.

The effects of projected daily mean temperatures above the current observed range were estimated using Monte Carlo simulation ( $n=$ $1000)[35,40]$. Estimated hospitalisation costs were also adjusted for future population estimates in the 2030s and 2050s as per ABS projections [25].

All statistical analyses were performed using R software (version 4.1.0) with the packages "dlnm", "tsModel" and function "attrdl" [35, 41]. The statistical significance level of 0.05 was adopted for the analyses.

\section{Results}

\subsection{Descriptive results of daily temperatures and hospitalization costs}

Table 1 shows the daily temperatures, hospital admissions for respiratory diseases and associated hospitalization costs in Sydney and Perth for the baseline study period. The daily mean temperatures in the two cities are quite similar with $18.1^{\circ} \mathrm{C}$ in Sydney and 18.9 ${ }^{\circ} \mathrm{C}$ in Perth over the study period of 2010-2016. In Sydney there were 280,882 respiratory disease hospital admissions with an associated 2,080.3 million AUD in hospitalization costs (6,406 AUD per admission); in Perth there were 75,801 respiratory hospital admissions, with 709.3 million AUD in costs equating to 8,241 AUD per admission. Figure 1 shows a time series plot of daily mean temperatures and hospitalization costs for respiratory diseases in Sydney and Perth over the study period of 2010-2016, which may indicate an inverse relationship between daily mean temperatures and respiratory disease hospitalization costs. 
Table 1

Descriptive statistics of daily temperature data and daily hospitalization costs for respiratory diseases in Sydney and Perth, 2010-2016.

\begin{tabular}{|lll|}
\hline Characteristics & Sydney & Perth \\
\hline Daily mean temperature ${ }^{\circ} \mathrm{C}(\mathrm{SD})$ & $18.1(4.7)$ & $18.9(5.0)$ \\
\hline Respiratory hospital admissions & 280,882 & 75,801 \\
\hline Respiratory hospitalization costs & $2,080.3$ million AUD & 709.3 million AUD \\
\hline Cost per admission & 6,406 AUD & 8,241 AUD \\
\hline Observation days & 2192 & 2192 \\
\hline Population & 5.5 million & 2.1 million \\
\hline Climate & Temperate climate & Mediterranean climate \\
\hline Total hospital admissions & 356,683 & \\
\hline Total hospitalization costs & $2,789.6$ million AUD & \\
\hline SD: Standard Deviation & & \\
AUD: Australian dollars & & \\
\hline
\end{tabular}

\subsection{Exposure-response relationship between daily mean temperatures and hospitalization costs}

Figure 2 shows the overall cumulative exposure-response curves for daily mean temperatures and respiratory disease hospitalization costs in Sydney and Perth. The relationships between temperatures and respiratory disease hospitalization costs are presented as nonlinear associations. The effects of temperature on respiratory disease hospitalization costs were significantly higher at low temperatures than high temperatures in both cities, which is consistent with the implied relationship seen in Figure 1. Specifically, the risk increases slowly as temperatures decrease below the optimum temperature in Sydney, while there is a steeper increase in risk at lower temperatures in Perth, despite different optimum temperatures identified in the two cities. By contrast, only in Perth temperatures above the optimum show significant effects on hospitalization costs for respiratory diseases.

Figures 3 and 4 further present the lag-specific exposure-response curves of daily mean temperatures and respiratory disease hospitalization costs in Sydney and Perth. In Sydney, on days of low temperature exposure (Lag 0 days) respiratory hospitalization costs were significantly reduced relative to the optimum temperature. However, increased costs due to the lagged effects of low temperatures were evident from Lag 2-12 days. Exposure to higher temperatures in Sydney did not present a significant effect on the hospitalization costs. In Perth, lagged exposures (Lag 2-12 days) to low temperatures showed a similar pattern as in Sydney, with evidence of increased hospitalization costs for respiratory diseases at lower temperatures. In addition, exposure to high temperatures (Lag 0 day) significantly increased hospitalization costs in Perth.

\subsection{The effects of current and future temperature increase on hospitalization costs for respiratory diseases}

Table 2 reports the estimates of the total fraction of hospitalization costs for respiratory diseases due to temperature during the baseline study period of 2010 s. Overall, $23.71 \%$ (95\% Cl: $8.39-34.96)$ and $13.82 \%$ (95\% Cl: $5.95-20.90)$ of respiratory disease hospitalization costs were attributed to non-optimum temperatures in Sydney and Perth, respectively, in 2010s. The total fraction can be separated into two components due to cold and hot temperatures. The comparison of the two components shows that cold temperatures are responsible for most respiratory disease hospitalization costs with $23.69 \%$ (95\% Cl: 8.28-34.99) and 11.20\% (95\% Cl: 2.93-18.67), compared to 0.02\% (95\% Cl: -0.08-0.11) and 2.64\% (95\% Cl: 0.65-4.72) for hot temperatures in Sydney and Perth, respectively. Despite the larger component attributable to temperatures in Sydney, the contribution from hot temperatures is significant in Perth only. The results are consistent with the overall cumulative exposure-response curves in Figure 2.

Page 6/16 
For the estimation of future hospitalization costs for respiratory diseases under three RCP scenarios (RCP2.6, RCP4.5 and RCP8.5)

over the periods 2030 s and 2050s (Table 2), the estimated overall fraction attributable to temperature slightly reduced from $23.71 \%$ in 2010 s to $20.26 \%, 20.07 \%$ and $19.67 \%$ under the RCP2.6, RCP4.5 and RCP8.5 scenarios, respectively for 2030s in Sydney. The estimates would be further reduced to $18.09 \%$ under high emission scenario RCP8.5 in 2050s. A similar pattern was evident for Perth, with the overall fraction attributable to temperature reducing from $13.82 \%$ in 2010 s to $11.34 \%-11.20 \%$ in 2030 s to $11.24 \%-10.54 \%$ in 2050s. Specifically, the estimated cold-attributable fraction of hospitalization costs for respiratory diseases would reduce from $20.24 \%-19.64 \%$ in 2030 s to $20.08 \%-18.04 \%$ in 2050 s in Sydney, and the cold-attributable fraction would reduce from $6.75 \%-6.46 \%$ in 2030 s to $6.63 \%-5.07 \%$ in 2050 s in Perth. By contrast, the heat-attributable fraction of hospitalization costs for respiratory diseases in Perth would slightly increase from $4.61 \%-4.76 \%$ in 2030 s to $4.63 \%-5.48 \%$ in 2050 s under the three different climate change scenarios, but in Sydney there is no statistically significant increase in the heat-attributable fraction. Nevertheless, it should be noted that the absolute costs attributed to temperature increased substantially from 493.2 million AUD in 2010s to more than 700 million AUD in 2050s in Sydney, and from 98.0 million AUD to around 150 million AUD during the same period in Perth.

The results of the sensitivity analyses can be found in supplementary materials. These analyses include changing the daily mean temperatures to daily minimum and maximum temperatures to capture the association between daily temperatures and hospitalization costs for respiratory diseases (Figure S2), the $d f$ of the B-spline for the calendar year from 4 to 8 per year (Figure S3), maximum lag days from 7 to 21 days (Figure S4), and residuals for the DLNM models (Figure S5). The residuals for the DLNM models followed a normal distribution, and no significant autocorrelations were found in the residuals. 
Table 2

Total fraction (\%) of hospitalization costs for respiratory diseases attributable to temperature, reported as overall, cold and heat components with $95 \%$ confidence intervals $(\mathrm{Cl})$.

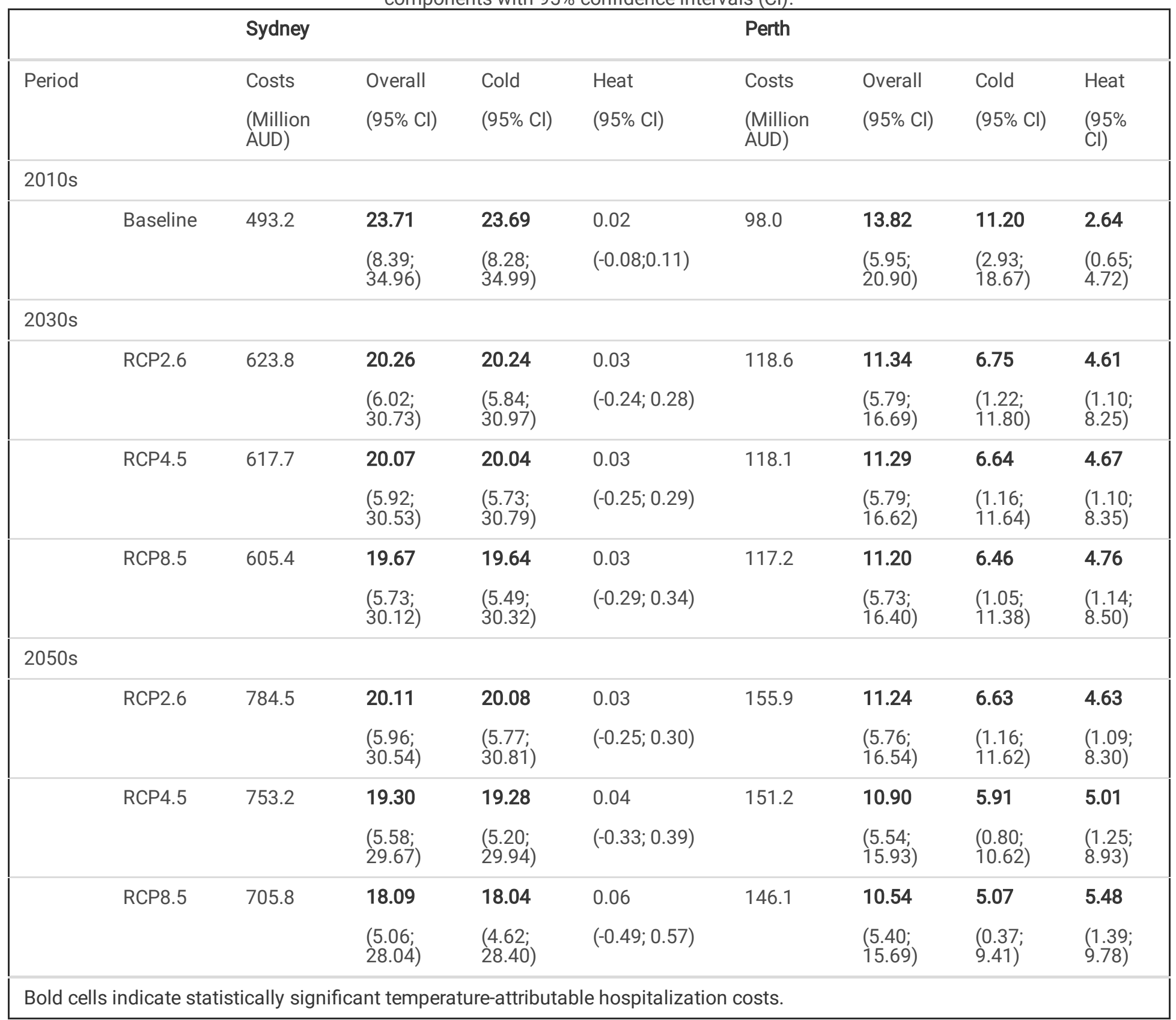

\section{Discussion}

This study presents results on the effects of temperature on hospitalization costs for respiratory diseases in two Australian cities with different climatic characteristics, and estimates the changes of such costs under different climate change scenarios in the future. We found that Sydney has a higher number of respiratory hospital admissions (280,882 admissions) compared with Perth (75,801 admissions), which could not be entirely explained by population size in the two cities ( 5.5 million and 2.1 million respectively). This could be due to Sydney being a larger city with a high-density population that may facilitate occurrence or transmission of certain types of respiratory diseases, e.g., asthma, flu and pneumonia, and different climatic and environmental characteristics [20, 42]. Moreover, the average cost per admission is lower in Sydney (6,406 AUD) relative to Perth (8,241 AUD), which could be due to the number of specific types of respiratory diseases and associated hospitalization costs that could be further explored in future studies.

We found there were overall non-linear relationships between ambient temperature exposures and respiratory disease hospitalization costs in Sydney and Perth. Both cold and hot temperatures can increase the hospitalization costs, with a significantly higher risk at lower temperatures in both cities. This is consistent with other studies exploring the impact of temperature on morbidity due to 
respiratory diseases, suggesting more cold-related respiratory diseases than heat-related respiratory diseases $[16,17,20]$. Furthermore, the lag0 day exposure to cold is significantly associated with reduced costs for respiratory disease hospitalizations in Sydney. This could be explained by limited human outdoor activities during sudden cold exposure and hence reduced cold exposure and associated costs for cold-related respiratory hospital admissions; or patients may seek medical care from emergency department for acute onset conditions such as asthma that was not accounted into hospitalization costs. However, exposure to cold temperatures from 2 days to 12 days is associated with an increase in hospitalization costs for respiratory diseases. Previous studies also showed the delayed cold effect on respiratory diseases which could persist up to a longer lag of 2 weeks or more [43,44]. In Perth, the trend with reduced hospitalization costs on lag0 day is also apparent, although it was not significant.

Further, we found an acute heat effect on hospitalization costs on the day of heat exposure in Perth, but not in Sydney. The observed differences between the two cities could be due to the different climates and population adaptation. The optimum temperatures identified in Sydney where it has a higher relative humidity across the year, was $27.7^{\circ} \mathrm{C}$, and this temperature is plausible for people living in higher relative humid areas as the higher temperature is needed for heat dissipation. In Perth where it has a dry climate, the optimum temperature was $20.6^{\circ} \mathrm{C}$. Residents in Sydney might acclimate to the temperate climate with warm summers but susceptible to cold temperatures, whilst the residents in Perth were less likely to acclimate to the Mediterranean climate of hot and dry summers, and were vulnerable to both hot summer and cold winter. Moreover, more rainfall in the summer of Sydney may reduce personal exposure to hot temperatures [45]. The acclimatization potentially modifies the optimum temperatures for the two cities and the magnitude of temperature effect on the hospitalization costs for respiratory diseases. Nevertheless, the two optimum temperatures are generally in accordance with those reported in other studies worldwide that indicated a thermal comfort range of $16.5^{\circ} \mathrm{C}$ to $29^{\circ} \mathrm{C}$ for human health $[36,46]$.

The study estimated 493.2 million AUD and 98.0 million AUD hospitalization costs for respiratory diseases associated with ambient temperature exposures over the baseline study period in Sydney and Perth, respectively. The cold attributable fraction was as high as $23.7 \%$ in Sydney, compared with $11.2 \%$ in Perth. The results for both cities indicated that most of the hospitalization costs for respiratory diseases were attributed to cold temperatures. The possible explanatory mechanism could be that cold temperatures are more likely to increase the human physiological susceptibility to respiratory diseases [47]. Acute chilling and cold temperatures can increase respiratory diseases $[48,49]$. Laboratory evidence has suggested that inhalation of cold air, cooling the body temperature, may cause pathophysiological responses that affect the immune system and increase the susceptibility to respiratory diseases $[14,15$, 50]. Further, cold weather can lead to more wood-fired heaters at homes and generate particulate matter in enclosed environments which might be linked to respiratory conditions. Other reasons could include that people may stay indoors and crowd together during wintertime, and facilitate virus spread or bacteria transmission and increase the incidence of disease [47]. These would in turn increase the economic burden to healthcare due to respiratory diseases when the temperature is lower. By contrast, the heat attributable fractions in the two cities were much smaller. The comparison between cold and heat attributable fractions for hospitalization costs indicates that the burden of respiratory disease due to heat in Sydney and Perth is lower relative to the burden during the cold winter period, which is consistent with other studies $[16,17,20,44]$.

The estimation of the future hospitalization costs attributable to temperature for respiratory diseases showed a substantial increase from about 500 million AUD and 100 million AUD at the baseline period in Sydney and Perth, respectively, to approximately 700 million AUD and 150 million AUD by midcentury, despite its declining attributable fractions to non-optimum temperature. The possible explanation could be the future burgeoning population by the middle of this century up to 9.1 million population in Sydney and 3.7 million population in Perth [25]. In addition, the significantly increasing aging population in Australia would also be more susceptible to respiratory diseases $[47,51]$. Thus, the absolute hospitalization costs will be increased substantially. Furthermore, it is worthy to notice that the heat attributable fraction for respiratory diseases was estimated to double in Perth from $2.64 \%$ in 2010 s up to $5.48 \%$ in 2050 s. This indicates that close attention should be paid to heat-related respiratory diseases in the future. In the context of climate change, more extreme weather events such as heatwaves, bushfires, droughts, dust storms, longer pollen seasons and air pollution would further deteriorate respiratory health and conditions, such as asthma, respiratory tract infections and chronic obstructive pulmonary disease, and increase the associated hospitalization costs in the future $[52,53]$.

This study is the first to examine the temperature effects on hospitalization costs for respiratory diseases over a long time period in Australia. We employed a DLNM model to control for confounding effects including seasonality, long-term trends, day-of-week and public holidays. We estimated both cold and hot temperature attributable hospitalization costs for respiratory diseases in cities with different climates and demographics. We also projected such hospitalization costs in the future in the context of climate change. In 
addition, the comparison between temperature effects in Sydney and Perth may suggest an acclimatization effect. The observed heterogeneity of the temperature effect on hospitalization costs for respiratory diseases may also indicate a need to conduct locationspecific and disease-specific studies to explore the temperature effect on population health outcomes in future.

Several limitations of this study should be acknowledged. First, we assumed the baseline exposure-response relationships for the estimation of future respiratory disease hospitalization costs would not change under different climate change scenarios in the future. However, human behavioural adaptations to changing climate in the long term may play a certain role in mitigating the effect of temperature on respiratory diseases. This may change the impact of temperature changes on respiratory disease hospitalization costs. Second, this study did not consider individual-level exposures, personal indoor and outdoor daily activity patterns, local air pollution levels, or smoking and other behavioural risks, which may alter the temperature effect on hospitalization costs for respiratory diseases. Third, the hospitalization costs for the respiratory disease may reflect only severe and acute episodes, which may underestimate the overall burden including those who attended the emergency department, and costs due to lost productivity for days off work. Fourth, the hospitalization costs were adjusted based on the consumer price index up to the second quarter of 2016, which may not reflect the future healthcare costs due to economic fluctuations including inflation, deflation or discounted cash flow. Lastly, the respiratory disease hospitalization costs were gathered from two cities in Australia, which may limit the generalizability of research findings to other areas and regions. Subsequent studies to explore costs in sub-tropical and inland locations would be warranted. In addition, respiratory disease hospitalization costs due to emerging infectious diseases and pandemics such as COVID-19, avian flu, SARS, and MERS in the future may not be particularly temperature-sensitive and cannot be estimated.

\section{Conclusions}

Cold temperatures are associated with increased hospitalization costs for respiratory diseases with delayed effects in Sydney and Perth, and are responsible for most of the temperature-attributable economic burden of respiratory disease hospitalizations. Currently, about $23.7 \%$ and $11.2 \%$ of respiratory disease hospitalization costs are attributable to cold temperatures in Sydney and Perth, respectively. The overall hospitalization costs attributable to temperature will increase from current about 500 million AUD to 700 million AUD in Sydney, and 100 million AUD to 150 million AUD in Perth by midcentury. The cold attributable fraction is likely to slightly decline due to global warming, while the hospitalization costs for respiratory diseases attributed to heat are estimated to gradually increase in the future. The findings highlight the significant impact of cold temperatures on the economic burden of respiratory diseases currently in Australia, and also may suggest that overall temperature attributable respiratory disease hospitalization costs are likely to increase substantially in Australia in the context of climate change.

\section{Declarations}

\section{Appendix A}

Supplementary Tables and Figures

\section{Ethics approval}

This study obtained ethics approval from the Human Research Ethics Committee of the University of Adelaide (Approval No. ID33179).

\section{Consent for publication}

All authors have read and approved the manuscript and give consent for publication.

\section{Availability of data and materials}

The datasets used and analyzed are available from the corresponding author on reasonable request and after approval by the Independent Hospital Pricing Authority.

\section{Competing Interests}

The authors declare that they have no competing interests.

\section{Funding}


This study was supported by the National Health and Medical Research Council (Application ID: APP1145239).

\section{Authors' contributions}

MT analyzed and interpreted the data, and drafted the manuscript. BW and KD assisted with the data analysis. All authors revised and edited the manuscript. All authors reviewed the document and approved the final version of the manuscript.

\section{Acknowledgements}

We acknowledge the Independent Hospital Pricing Authority, the Australian Bureau of Meteorology and the Australian Bureau of Statistics for providing access to healthcare, meteorological and population statistics.

\section{References}

1. Schurer, A.P., Mann, M.E., Hawkins, E., Tett, S.F.B., Hegerl, G.C. Importance of the Pre-Industrial Baseline in Determining the Likelihood of Exceeding the Paris Limits. Nature climate change 2017, 7, 563-567.

2. Zhang, Y., Beggs, P.J., Bambrick, H., Berry, H.L., Linnenluecke, M.K., Trueck, S., et al. The MJA-Lancet Countdown on health and climate change: Australian policy inaction threatens lives. Med. J. Aust. 2018, 209, 474.

3. Butler, C.D. Climate Change, Health and Existential Risks to Civilization: A Comprehensive Review (1989(-)2013). Int. J. Environ. Res. Public. Health 2018, 15.

4. Ye, X., Wolff, R., Yu, W., Vaneckova, P., Pan, X., Tong, S. Ambient temperature and morbidity: a review of epidemiological evidence. Environ. Health Perspect. 2012, 120, 19-28.

5. Basu, R., Samet, J.M. Relation between Elevated Ambient Temperature and Mortality: A Review of the Epidemiologic Evidence. Epidemiol. Rev. 2002, 24, 190-202.

6. Williams, S., Nitschke, M., Weinstein, P., Pisaniello, D.L., Parton, K.A., Bi, P. The impact of summer temperatures and heatwaves on mortality and morbidity in Perth, Australia 1994-2008. Environment International 2012, 40, 33-38.

7. Borg, M., Bi, P., Nitschke, M., Williams, S., McDonald, S. The impact of daily temperature on renal disease incidence: an ecological study. Environ. Health 2017, 16, 114.

8. Xiang, J., Hansen, A., Liu, Q., Liu, X., Tong, M.X., Sun, Y., et al. Association between dengue fever incidence and meteorological factors in Guangzhou, China, 2005-2014. Environ. Res. 2016, 153, 17-26.

9. Xiang, J., Hansen, A., Liu, Q., Tong, M.X., Liu, X., Sun, Y., et al. Association between malaria incidence and meteorological factors: a multi-location study in China, 2005-2012. Epidemiol. Infect. 2017, 146, 89-99.

10. Xiang, J., Hansen, A., Liu, Q., Tong, M.X., Liu, X., Sun, Y., et al. Impact of meteorological factors on hemorrhagic fever with renal syndrome in 19 cities in China, 2005-2014. Sci. Total Environ. 2018, 636, 1249-1256.

11. Bi, P., Tong, S.L., Donald, K., Parton, K., Hobbs, J. Climate variability and the transmission of Ross River virus infection in the coastal region of Queensland, Australia. Epidemiology 2000, 11, S64-S64.

12. Watts, N., Amann, M., Ayeb-Karlsson, S., Belesova, K., Bouley, T., Boykoff, M., et al. The Lancet Countdown on health and climate change: from 25 years of inaction to a global transformation for public health. The Lancet 2017.

13. Kim, K.H., Kabir, E., Ara Jahan, S. A review of the consequences of global climate change on human health. J Environ Sci Health C Environ Carcinog Ecotoxicol Rev 2014, 32, 299-318.

14. Eccles, R., Wilkinson, J.E. Exposure to cold and acute upper respiratory tract infection. Rhinology 2015, 53, 99-106.

15. Mäkinen, T.M., Juvonen, R., Jokelainen, J., Harju, T.H., Peitso, A., Bloigu, A., et al. Cold temperature and low humidity are associated with increased occurrence of respiratory tract infections. Respir. Med. 2009, 103, 456-462.

16. Ma, W., Xu, X., Peng, L., Kan, H. Impact of extreme temperature on hospital admission in Shanghai, China. Sci. Total Environ. 2011, 409, 3634-3637.

17. Xu, Z., Hu, W., Su, H., Turner, L.R., Ye, X., Wang, J., et al. Extreme temperatures and paediatric emergency department admissions. J. Epidemiol. Community Health 2014, 68, 304-311.

18. Lin, S., Luo, M., Walker, R.J., Liu, X., Hwang, S.A., Chinery, R. Extreme high temperatures and hospital admissions for respiratory and cardiovascular diseases. Epidemiology 2009, 20, 738-746. 
19. Michelozzi, P., Accetta, G., De Sario, M., D'Ippoliti, D., Marino, C., Baccini, M., et al. High temperature and hospitalizations for cardiovascular and respiratory causes in 12 European cities. Am. J. Respir. Crit. Care Med. 2009, 179, 383-389.

20. Liu, Y., Guo, Y., Wang, C., Li, W., Lu, J., Shen, S., et al. Association between temperature change and outpatient visits for respiratory tract infections among children in Guangzhou, China. Int. J. Environ. Res. Public. Health 2015, 12, $439-454$.

21. Lin, Y.-K., Chang, C.-K., Chang, S.-C., Chen, P.-S., Lin, C., Wang, Y.-C. Temperature, nitrogen dioxide, circulating respiratory viruses and acute upper respiratory infections among children in Taipei, Taiwan: A population-based study. Environ. Res. 2013, 120, $109-118$.

22. Tong, M.X., Wondmagegn, B.Y., Xiang, J., Williams, S., Hansen, A., Dear, K., et al. Emergency department visits and associated healthcare costs attributable to increasing temperature in the context of climate change in Perth, Western Australia, 2012-2019. Environmental Research Letters 2021, 16, 065011.

23. Lin, S., Hsu, W.H., Van Zutphen, A.R., Saha, S., Luber, G., Hwang, S.A. Excessive heat and respiratory hospitalizations in New York State: estimating current and future public health burden related to climate change. Environ. Health Perspect. 2012, 120, 15711577.

24. CSIRO and Bureau of Meteorology. Climate Change in Australia. Projections for Australia's NRM Regions Available online: https://www.climatechangeinaustralia.gov.au/media/ccia/2.2/cms_page_media/168/CCIA_2015_NRM_TechnicalReport_WEB.pdf (accessed on 5 August 2015).

25. Australian Bureau of Statistics. Population Projections, Australia. Available online: https://www.abs.gov.au/AUSSTATS/abs@.nsf/DetailsPage/3222.02017\%20(base)\%20-\%202066?OpenDocument (accessed on 10 June 2018).

26. Australian Bureau of Statistics. Regional population Available online: https://www.abs.gov.au/statistics/people/population/regional-population/latest-release (accessed on 25 April 2021 ).

27. Commonwealth Scientific and Industrial Research Organisation. Climate Futures Exploration Tool. Available online: https://www.climatechangeinaustralia.gov.au/en/projections-tools/climate-futures-tool/detailed-projections/ (accessed on 11 June 2018).

28. Newbury, J. Linear Interpolation. In Basic Numeracy Skills and Practice, Macmillan Education UK: London, 1981, pp 67-72.

29. Tong, M.X., Wondmagegn, B.Y., Williams, S., Hansen, A., Dear, K., Pisaniello, D., et al. Hospital healthcare costs attributable to heat and future estimations in the context of climate change in Perth, Western Australia. Advances in Climate Change Research 2021, $12,638-648$.

30. Tong, M., Wondmagegn, B.Y., Xiang, J., Williams, S., Hansen, A., Dear, K., et al. Heat-attributable hospitalisation costs in Sydney: Current estimations and future projections in the context of climate change. Urban Climate 2021, 40, 101028.

31. Wondmagegn, B.Y., Xiang, J., Dear, K., Williams, S., Hansen, A., Pisaniello, D., et al. Increasing Impacts of Temperature on Hospital Admissions, Length of Stay, and Related Healthcare Costs in the Context of Climate Change in Adelaide, South Australia. Sci. Total Environ. 2021, 145656.

32. Gasparrini, A., Armstrong, B., Kenward, M.G. Distributed lag non-linear models. Stat. Med. 2010, $29,2224-2234$.

33. Gasparrini, A. Distributed Lag Linear and Non-Linear Models in R: The Package dlnm. J Stat Softw 2011, 43, 1-20.

34. Gasparrini, A. Statistical Methods in Studies on Temperature-health Associations. London School of Hygiene and Tropical Medicine (University of London), 2011.

35. Gasparrini, A., Leone, M. Attributable risk from distributed lag models. BMC Med. Res. Methodol. $2014,14,55$.

36. Gasparrini, A., Guo, Y., Hashizume, M., Lavigne, E., Zanobetti, A., Schwartz, J., et al. Mortality risk attributable to high and low ambient temperature: a multicountry observational study. The Lancet 2015, 386, 369-375.

37. Gasparrini, A. Modeling exposure-lag-response associations with distributed lag non-linear models. Stat. Med. 2014, 33, 881899.

38. Bai, L., Cirendunzhu, Woodward, A., Dawa, Zhaxisangmu, Chen, B., et al. Temperature, hospital admissions and emergency room visits in Lhasa, Tibet: A time-series analysis. Sci. Total Environ. 2014, 490, 838-848.

39. Australian Bureau of Statistics. Consumer Price Index, Australia. Available online: https://www.abs.gov.au/statistics/economy/price-indexes-and-inflation/consumer-price-index-australia/latest-release (accessed on 30 September 2020).

40. Vicedo-Cabrera, A.M., Sera, F., Gasparrini, A. Hands-on tutorial on a modeling framework for projections of climate change impacts on health. Epidemiology 2019, 30, 321-329.

Page 12/16 
41. R Core Team. R: A language and environment for statistical computing. 2013.

42. Buckley, J.P., Richardson, D.B. Seasonal modification of the association between temperature and adult emergency department visits for asthma: a case-crossover study. Environ. Health 2012, 11, 55.

43. Analitis, A., Katsouyanni, K., Biggeri, A., Baccini, M., Forsberg, B., Bisanti, L., et al. Effects of cold weather on mortality: results from 15 European cities within the PHEWE project. Am. J. Epidemiol. 2008, 168, 1397-1408.

44. Zhao, Q., Zhao, Y., Li, S., Zhang, Y., Wang, Q., Zhang, H., et al. Impact of ambient temperature on clinical visits for cardio-respiratory diseases in rural villages in northwest China. Sci. Total Environ. 2018, 612, 379-385.

45. Australian Bureau of Meteorology. Monthly Rainfall Available online: http://www.bom.gov.au/climate/data/index.shtml (accessed on 12 July 2021).

46. Patz, J.A., Campbell-Lendrum, D., Holloway, T., Foley, J.A. Impact of regional climate change on human health. Nature 2005, 438, 310-317.

47. Eccles, R. An explanation for the seasonality of acute upper respiratory tract viral infections. Acta Otolaryngol 2002, $122,183-191$.

48. Johnson, C., Eccles, R. Acute cooling of the feet and the onset of common cold symptoms. Fam. Pract. 2005, 22, 608-613.

49. Lowen, A.C., Mubareka, S., Steel, J., Palese, P. Influenza virus transmission is dependent on relative humidity and temperature. PLoS Pathog. 2007, 3, 1470-1476.

50. Mourtzoukou, E.G., Falagas, M.E. Exposure to cold and respiratory tract infections. Int. J. Tuberc. Lung Dis. 2007, 11, 938-943.

51. Australian Institute of Health and Welfare. Older Australia at a glance. Available online: https://www.aihw.gov.au/reports/olderpeople/older-australia-at-a-glance/contents/demographics-of-older-australians/australia-s-changing-age-and-gender-profile (accessed on 15 August 2021).

52. Anderson, G.B., Dominici, F., Wang, Y., McCormack, M.C., Bell, M.L., Peng, R.D. Heat-related emergency hospitalizations for respiratory diseases in the Medicare population. Am. J. Respir. Crit. Care Med. 2013, 187, 1098-1103.

53. Nairn, J., Beaty, M., Varghese, B.M. Australia's black summer heatwave impacts. Australian Emergency Management Institute: 2021, Vol. 36, p 17-20.

\section{Figures}



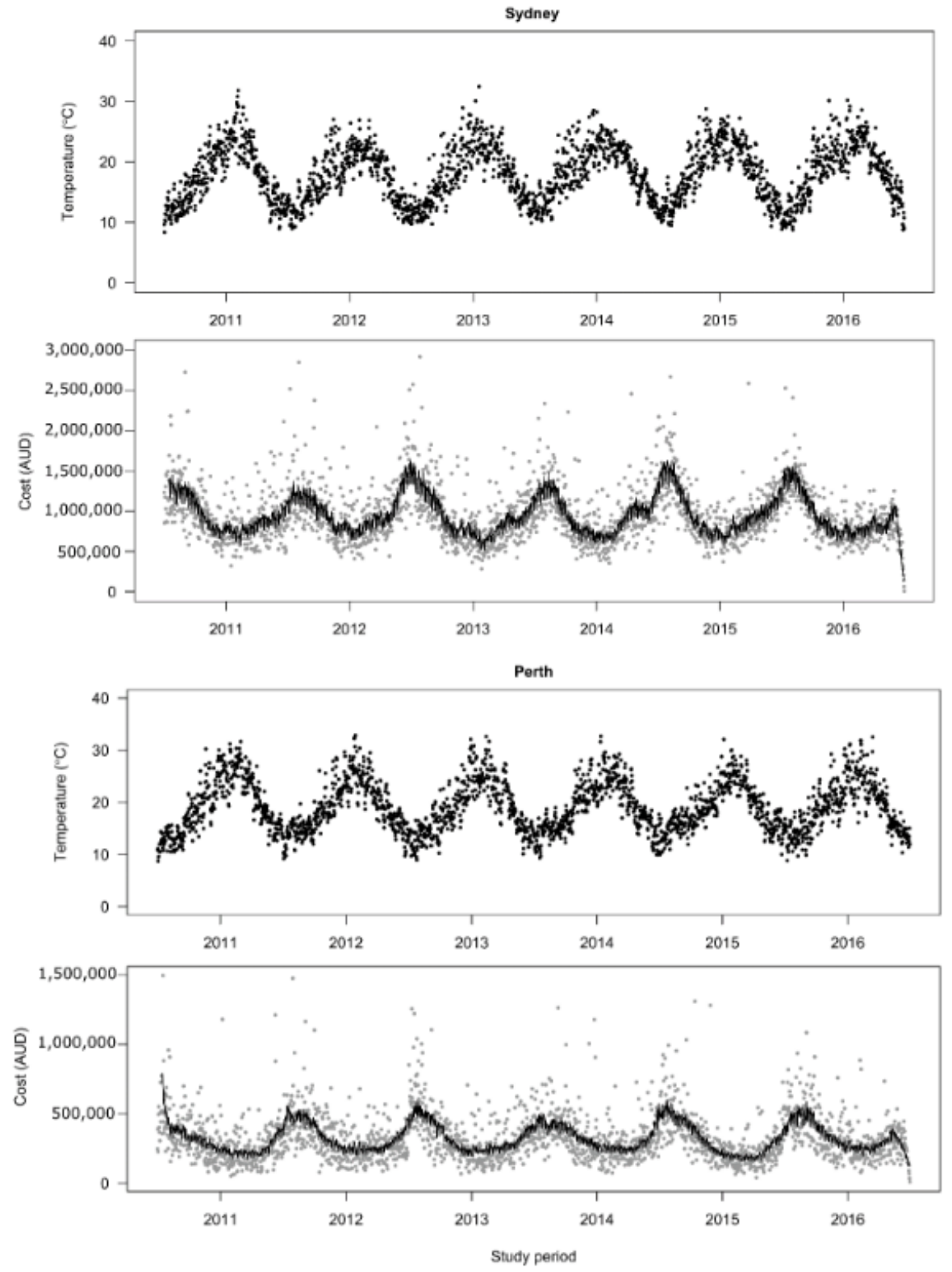

Figure 1

Time-series plots for daily mean temperatures and daily hospitalization costs of respiratory diseases in Sydney and Perth, 20102016.
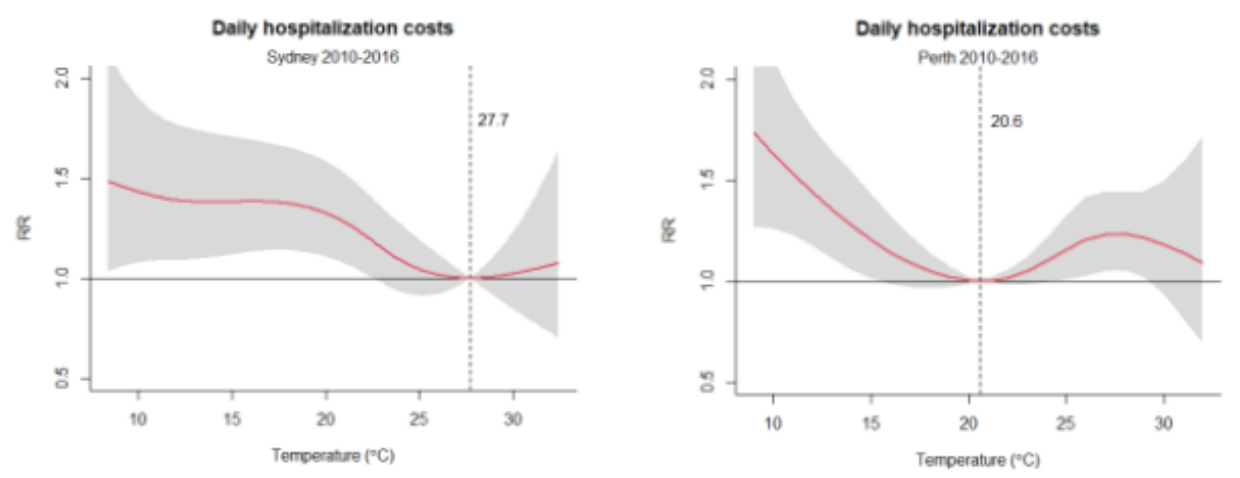

Figure 2 
Overall cumulative exposure-response relationships between daily mean temperatures and daily hospitalization costs for 14 lag days in Sydney and Perth, 2010-2016. RR is the relative risk for hospitalization costs. The optimum temperatures are $27.7^{\circ} \mathrm{C}$ and $20.6^{\circ} \mathrm{C}$ for respiratory disease hospitalization costs in Sydney and Perth, respectively.

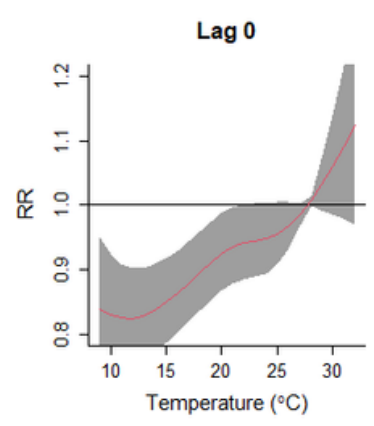

Lag 1



Lag 2

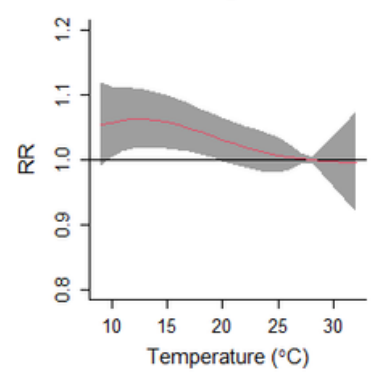

Lag 3



Lag 4

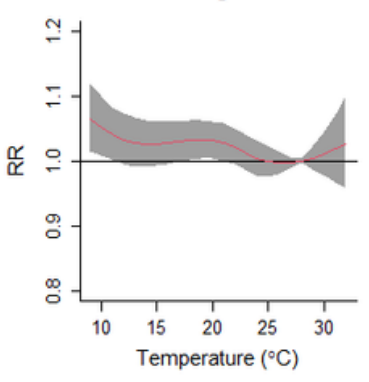

Lag 5

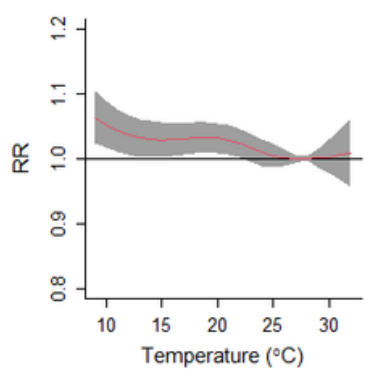

Lag 6



Lag 7

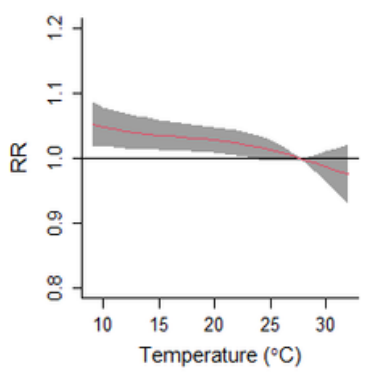

Lag 8

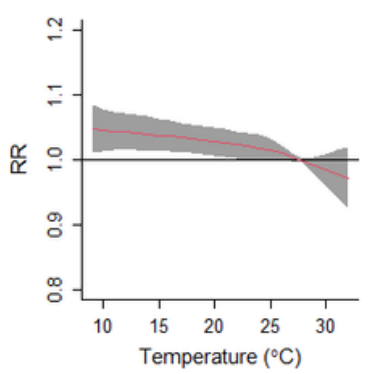

Lag 9

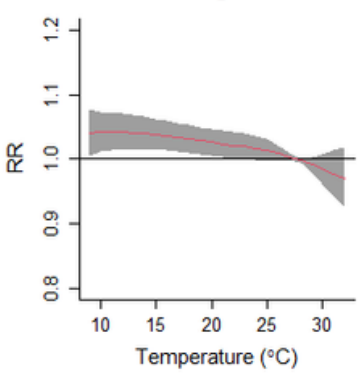

Lag 10

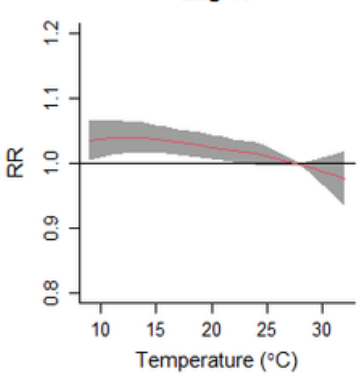

Lag 11

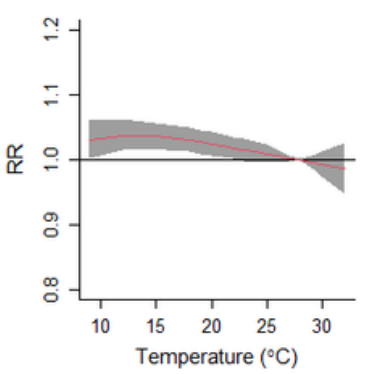

Lag 12

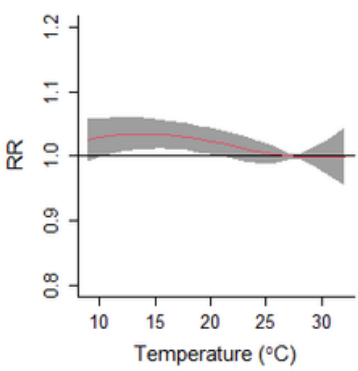

Lag 13

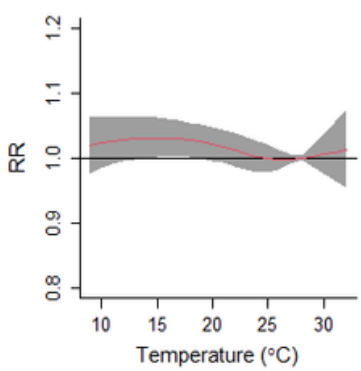

Lag 14

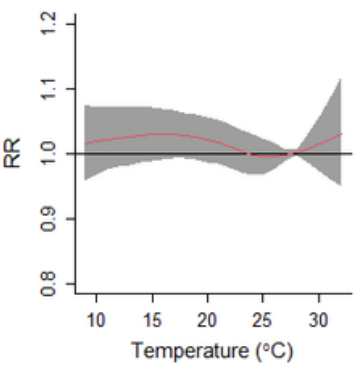

\section{Figure 3}

Lag-specific exposure-response curves for daily mean temperatures and hospitalization costs in Sydney, 2010-2016. RR is relative risk for respiratory disease hospitalization costs. 


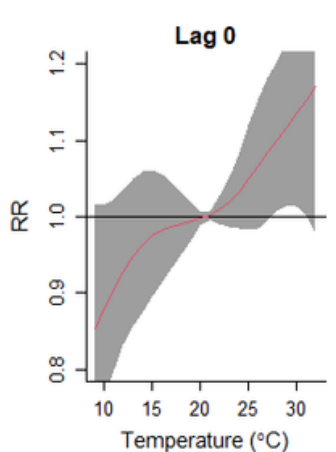

Lag 1

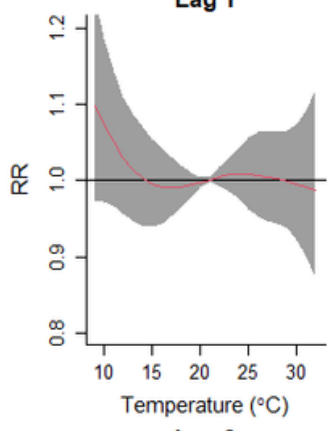

Lag 2

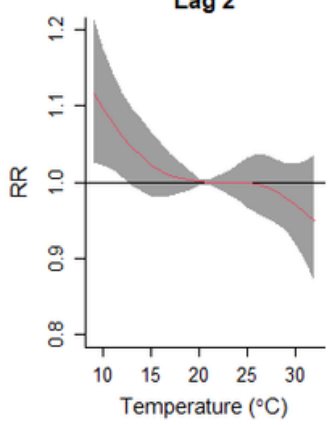

Lag 3

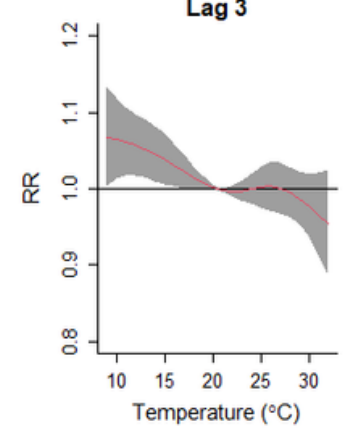

Lag 4

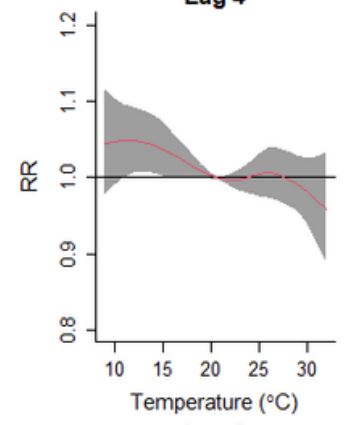

Lag 5

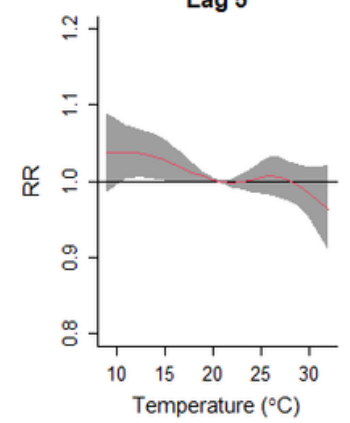

Lag 6

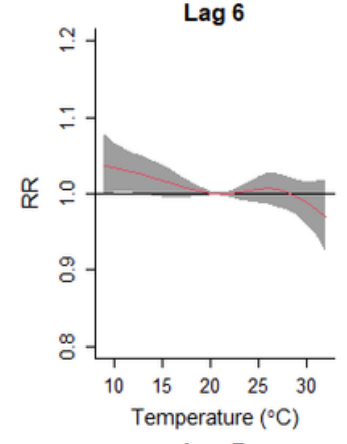

Lag 7
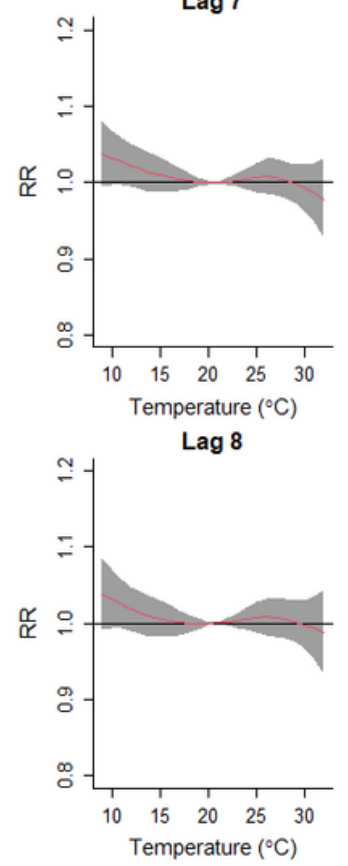

Lag 9

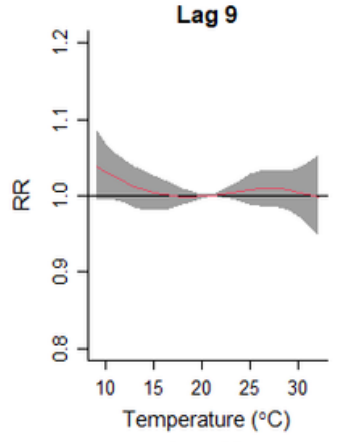

Lag 10
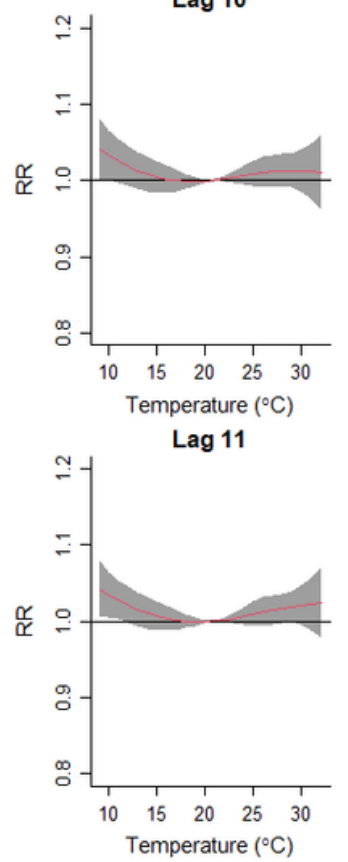

Lag 12

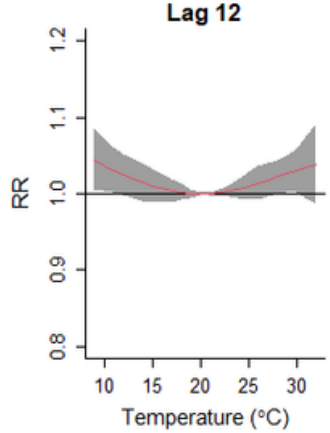

Lag 13

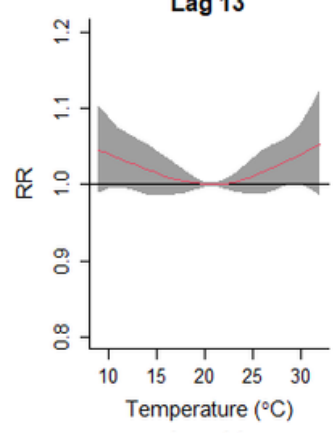

Lag 14

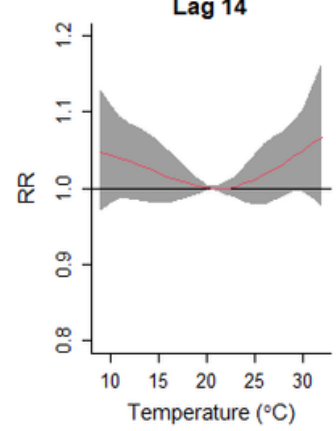

Figure 4

Lag-specific exposure-response curves for daily mean temperatures and hospitalization costs in Perth, 2010-2016. RR is relative risk for respiratory disease hospitalization costs.

\section{Supplementary Files}

This is a list of supplementary files associated with this preprint. Click to download.

- 4Supplementarymaterials.docx 